\title{
Packing defects and the width of biopolymer bundles
}

\author{
Nir S. Gov \\ Department of Chemical Physics, \\ The Weizmann Institute of Science, \\ P.O.B. 26, Rehovot, Israel 76100
}

\begin{abstract}
The formation of bundles composed of actin filaments and cross-linking proteins is an essential process in the maintenance of the cells' cytoskeleton. It has also been recreated by in-vitro experiments, where actin networks are routinely produced to mimic and study the cellular structures. It has long been observed that these bundles seem to have a well defined width distribution, which has not been adequately described theoretically. We propose here that packing defects of the filaments, quenched and random, contribute an effective repulsion that counters the cross-linking adhesion energy and leads to a well defined bundle width. This is a two-dimensional strain-field version of the classic Rayleigh instability of charged droplets.
\end{abstract}

PACS numbers:

Filamentous biopolymers, such as F-actin, have the ability to cross-link into a variety of bundles and networks, forming the cytoskeleton. The distribution of the radii of cross-linked actin bundles is a basic characteristic that determines the mechanical properties of the cytoskeleton. The thickness of bundles similarly determines the mechanical properties of artificial networks that form in-vitro [1, 2]. Recent in-vitro experiments 11] indicate a broad distribution of radii, with a distinct peak, while simple equilibrium theory would predict a global phase separation and formation of a single bundle of infinite width and length [4] (in an infinite system). Several possibilities have been proposed to explain the observed distribution; When the bundles form due to multi-valent ions, electrostatic interactions are unbalanced and can lead to a selection of an equilibrium finite radius [6]. A recent study attributes the finite size of the actin bundles to the inherent chirality of these filaments [7], and indeed this may be the dominant effect when the filaments are linked by small multi-valent ions [5, [8]. We will deal here with neutral systems that are strongly chemically cross-linked by larger linker proteins [2, 3, 11]. In these cases the chirality of the individual filaments may play a lesser role, and we will treat the filaments as achiral. Our model proposes therefore a complimentary mechanism for finite bundle widths, which applies to achiral bundles. We present here a model where quenched disorder, in the form of twist-defects (Fig.1), leads to the selection of a quasi-equilibrium finite radius. We call this a quasi-equilibrium since the defects in the bundle can be annealed away in principle, but this is highly unlikely for long filaments and strong cross-linking, and the system is therefore dynamically arrested in a meta-stable configuration. There is recent experimental evidence that in-vitro actin bundles do indeed have such twist defects [3]. We wish to consider the implication of such defects for the width distribution of in-vitro actin bundles. We will consider these knots to be in a form of quenched, i.e. static, and random disorder.

Consider a bundle of cross-linked filaments, as shown in Fig.1. The filaments are assumed to be tightly cross- linked to all their neighbors, so that they form a solid structure. If all the filaments are perfectly aligned then they form a hexagonal close-packed crystal. But in the process of aggregation twist-defects can form, where two filaments attach to the growing bundle in a different relative orientation along their lengths (Fig.1a). If these two filaments stay on the surface of the bundle and have a single such twist along their length, then this twist can slide to the ends of the filaments and relax away. When the filaments are long enough it is likely that they each form such twist-defects with other neighboring filaments in other places along their lengths, and the whole bundle is in fact knotted (Fig.1a). The knots can not be easily relaxed now by sliding them to the end of the filaments, as they are all entangled. The meandering of the filaments in the bundle due to the twist-defects may be described as a random walk of the filament, where the average number of defects along its length is: $N_{d e f} \sim\left(L / L_{p}\right) P_{d e f}$, where $L$ is the overall length of the filament, and each twistdefect extends over a length $L_{p}$ which is of the order of the persistence length of actin in the bundle $\left(L_{p} \sim 16 \mu \mathrm{m}\right.$ for free actin filaments [13]), and $P_{\text {def }}$ is the probability of exciting a defect.

When actin bundles attract each other, through the strong adhesion of cross-linking proteins, they begin to aggregate laterally. We will consider here the case of strong cross-linking and long filaments, so that the interaction term dominates over the entropy term in the free energy, which we will neglect from now on [6]. The adhesion energy gain is driving the aggregation, and since the filaments on the surface have less adhesion energy (less neighbors), there is an energetic drive to increase the bundle thickness. The adhesion energy per unit length can be simply written as

$$
E_{\text {bind }} \approx-\pi(R / a) \varepsilon_{b}((R / a)-2)
$$

where $\varepsilon_{b}$ is the adhesion energy per unit length, between the filaments due to the presence of the cross-linking proteins. The first term represents the bulk adhesion energy (negative) and the second term represents the surface energy (positive). This energy functional gives a critical 
radius of $R=a$ ( $a$ is the radius of the individual filaments and surrounding cross-linkers, which is typically [3, 11] 10nm), beyond which the bundle grows to infinite width. The observed widths of actin bundles seem to be rather well-defined [3], and despite the mobility of the filaments the bundles only grow by longitudinal aggregation and do not continue to thicken through lateral aggregation. This observation is therefore at odds with the equilibrium theory described by Eq.(10), and is our motivation for looking at the effect of packing defects.

We now treat the case of twist-defects inside the bundle. Each defect involves an increase in the local energy due to several terms (Fig.1): (i) Loss of adhesion energy due to broken cross-linking bonds at the site of the twist, (ii) the elastic energy of the twisted actin filaments, (iii) the elastic energy of the deformed hexagonal lattice of filaments around the defect in the twist plane (Fig.1). Out of these energies the first and third can be different if the defect is inside the bulk of the bundle or close to the surface. Furthermore, it is clear that these two terms are in fact smaller at the surface, so the energetic cost of a filament is higher inside the bulk of the bundle. A uniform distribution of static defects will therefore cost a higher energy the thicker is the bundle. We can already see how the defects may compete with the adhesion energy and drive the bundles to a finite preferred width.

Let us treat the bundle as being approximately uniform along its length, so that we can treat the defects in a twodimensional circular cross-section. The strain field in the surrounding bundle due to a localized defect depends on the order of the defect, i.e. a monopole or a higher multipole, and is maximal in the maximal twist-plane (Fig.1). A twist of two filaments increases the local volume of the filament packing (Fig.1), so has a monopole component (dilation). At the same time the twist involves also a quadropole component, as shown in Fig.1c. The single isolated monopole defect gives rise to a strain field that decays as $u \sim 1 / r$ away from the defect, and the resulting strain energy has a logarithmic divergence with the radius of the bundle. The contribution from such a single defect is therefore negligible compared to the $R^{2}$ term in Eq.(11), and the equilibrium stays at an infinite width.

We are therefore led to consider a uniform distribution of many defects. Before dealing with the complications of the long-range strain field of the monopole, let us treat the case of a highly localized strain-field, such as for the quadropole. In this case we can treat the strain energy in the bundle around the defect as part of the defect core energy $E_{c}$, that includes the broken cross-linker bonds and twist of the actin filaments.

A uniform distribution of defects, of density $\rho=1 / L^{2}$, will simply add $E_{\text {defect }}=\rho \pi R^{2} E_{c}$ to the energy of Eq.(1), without any qualitative change, i.e. the system still has minimum energy for an infinitely wide bundle. Let us assume that close to the rim of the bundle defects are not strongly trapped, and can relax. Such a process may occur due to higher mobility of the filaments on the bundle surface, which leads to effective "surface-melting" of several layers of filaments, thereby annealing any defects. The thickness of this annealed layer is denoted by $\lambda$ (Fig.2a). The energy of the defects (per unit length) in this system is therefore given by

$$
E_{\text {defect }} \approx \rho \pi(R-\lambda)^{2} E_{c}
$$

The total energy per unit length is now $E_{\text {total }}=$ $E_{\text {bind }}+E_{\text {defect }}$ (Eqs 112). We find that there is a critical value of the defect energy $E_{c}^{*}=\varepsilon_{b}(L / a)^{2}$, above which the system has an equilibrium configuration of finite width, given by

$$
R_{0, \text { shell }}=\left(E_{c} \lambda-a E_{c}^{*}\right) /\left(E_{c}-E_{c}^{*}\right)
$$

Note that as the defect core energy increases above its critical value, the bundle radius shrinks, for a fixed density. There is also a critical value of the width of the annealed layer, given by: $\lambda_{c}=a E_{c}^{*} / E_{c}$, at which the equilibrium bundle size shrinks to zero. Above $E_{c}^{*}$ the system is dominated by the energy of the defects, while below it the binding energy dominates. In the limit of $E_{c} \gg \varepsilon_{b}, E_{c}^{*}$, we find that the equilibrium radius approaches the thickness of the defect-free layer $R_{0, \text { shell }} \rightarrow \lambda$, while in the limit $E_{c} \rightarrow E_{c}^{*}$ the equilibrium radius diverges.

When the width of the defect-free outer shell is given by the typical inter-defect distance, i.e. $\lambda=L$, we get an equilibrium configuration of finite width, given by (Eq 3): $R_{0, \text { shell }}=L\left(E_{c}-\varepsilon_{b} L / a\right) /\left(E_{c}-E_{c}^{*}\right)$, and a critical interdefect separation of: $L_{c}=a\left(E_{c} / \varepsilon_{b}\right)$.

We now return to the problem of a uniform distribution of strain-monopoles. These can be treated as a twodimensional gas of charges, all of the same sign, interacting via logarithmic repulsion [10] (Fig.2b, bottom). Note that since the twist-defects cause a local dilation, they all have the sam sign and repel each other. The problem therefore resembles a two-dimensional version of the famous Rayleigh-instability of charged droplets [12]. In our case the twist-defects behave as elastic charges and their mutual repulsion breaks the infinitely thick bundle into bundles of finite radius.

We can estimate the overall strain energy (per unit length) of the system of uniform monopoles by calculating the interaction of a central charge with a uniform distribution of surrounding charges, assuming that its nearest neighbor is a distance $L$ away (Fig.2b)

$$
\begin{gathered}
E_{\text {mon }} \approx k \ln (L / a)+2 k \rho \int_{L}^{R} \ln (r / a) d^{2} r \\
=k \ln (L / a)+2 k \pi\left(1-\frac{R^{2}}{L^{2}}-\ln (L / a)+\frac{R^{2}}{L^{2}} \ln (R / a)\right)
\end{gathered}
$$

where $k$ is the elastic modulus of the bundle which is of order $\varepsilon_{b}$. The first term in Eq. (44) is the energy to create the defect at a distance $L$ from its nearest neighbor, and the second is the interaction energy with a uniform distribution of surrounding defects. We find that the dominant term in $R$ is of order $(R / L)^{2} \ln (R / a)$. Since we do not 
attempt an exact solution of the complex strain-field inside a cylindrical bundle with defects, we will continue with a scaling analysis using this term, i.e. we will approximate the strain-field energy per unit length due to the defects as

$$
E_{\text {defects }} \approx 2 \pi k^{\prime} \frac{R^{2}}{L^{2}} \ln (R / a)
$$

where $k^{\prime}$ combines the effective stiffness of the bundle and various geometric factors. This energy dominates at large $R$ over the binding energy (Eq11), due to the $\ln (R / a)$ factor. We therefore have a situation now where the bundles always have a finite equilibrium radius.

The total energy per unit length is now $E_{\text {total }}=$ $E_{\text {bind }}+E_{\text {defects }}$ (Eqs 15), and has a global minimum at the equilibrium radius, given by

$$
R_{0, \text { mono }} / a=\exp \left(\left(1 / 2 a^{2} \rho \alpha\right)-1 / 2\right)
$$

where $\alpha \equiv k^{\prime} / \varepsilon_{b}$. We find that as the density of defect increases the radius decreases, while it also decreases with the stiffness of the bundle $\alpha$. Note that the equilibrium radius has an exponential dependence on the density of defects, and is therefore predicted to be very sensitive to this parameter. The calculation we have given above are all appropriate for a system with an infinite reservoir of actin filaments and cross-linking proteins. In a closed system with a finite number of available filaments and cross-linkers we have to conserve the overall area of actin bundles, which should not change the overall behavior we described.

If the defects are interacting strongly with each-other through their quadropole component of the strain, then they can arrange in string-like aggregates (Fig.1e), in the maximal twist-plane. These aggregates can form if the density of defects is high and they are mobile enough to move inside the bundle and aggregate. Alternatively such aggregates of defects may form as the filaments join the bundle at the surface, since a defect is more likely to form close to an existing defect; at the right orientation it has a lower strain energy (Fig.1d). Such aggregations of defects will modify the strain-field and the contribution of the defect energy, but is beyond the scope of this paper.

Let us now compare our calculation with recent invitro measurements of the distribution of actin bundle radii [11]. The size distribution of bundles can be approximated to follow from a simple Boltzman distribution of the bundle energy: $P(R) \propto$ $\exp \left(-\left(E_{\text {total }}(R)-E_{0}\right) / k_{B} T\right.$. Since the bundle energy has a quadratic minimum at $R_{0}$ (Eqs 36), this gives a Gaussian distribution of radii centered around the equilibrium radius. The distribution of the number of actin filaments inside the bundles $N$ is simply the distribution of bundle areas, $N \propto(R / a)^{2}$, which we we predict to have roughly an exponential behavior at large $N$ since both Eqs. 225) are quadratic with $R$ (for the monopoles there is a slight logarithmic correction). This is in good agreement with the observed distribution (Fig.3).
The width of the distribution can be estimated from the above calculation, using

$$
\begin{aligned}
\left\langle\Delta R^{2}\right\rangle & =\left.\frac{k_{B} T}{\partial^{2} E_{\text {total }} / \partial R^{2}}\right|_{R_{0}} \\
& =\frac{k_{B} T}{2 \rho\left(E_{c}-E_{c}^{*}\right)}, \quad \frac{k_{B} T}{4 k^{\prime} \rho}
\end{aligned}
$$

where the first is for the empty shell case (Eqs 23), and the second is for the uniform field of monopoles (Eqs 56). We find that as the density of defects increases the width of the bundle sizes distribution decreases, and the distribution becomes tighter around $R_{0}$.

In Fig.3 we plot the calculated distribution compared to the experimental measurement [11], at a ratio of 1:5 Fascin cross-linkers to actin in the solution. In these fits we have to choose the unknown binding energy, and then fit the value of the parameters so that we get the position of the peak of the distribution and its width (Eq17). There is some freedom in the choice of these parameters; for the empty-shell model we choose the values; $\varepsilon_{b}=10 k_{B} T / a$ which is of the order of the known adhesion energy per typical actin cross-linker [4], and $a^{2} \rho=0.04$ which corresponds to a rather dense array of defects and is quite arbitrarily. By fitting to the experimental distribution, we then fix the values of $\lambda / a=1.007$ and $E_{c}=250 k_{B} T / a$.

For the uniform-monopoles the width of the observed distribution fixes the value of $a^{2} \rho k^{\prime}$, and we take $a^{2} \rho=$ 0.1 and $k^{\prime}=0.03 k_{B} T / a$, which then fixes the value of $\varepsilon_{b}=0.05 k_{B} T / a$, by fitting to the peak location. We find that for a good fit we need a very small value for the binding energy per unit length. The reason for this may arise from the fact that our two-dimensional monopoles represent twist-defects which extends over a length of the order of the persistence length of actin $L_{p}$ [13]. This may be much longer than the effective thickness of the maximal twist-plane $L_{\text {mono }}$ (Fig. 1$)$. If $L_{\text {mono }} \sim a$ then all the energy terms are re-scaled by a factor of $L_{\text {mono }} / L_{p} \sim$ $10^{-2}-10^{-3}$.

It has been further observed that the average thickness of the bundles increases with the concentration of crosslinking proteins [2, 11]. Within our model the radius of the peak of the distribution, at $R_{0, \text { shell }}$ or $R_{0, \text { mono }}$, depends only on the density of defects $\rho$ if both the binding energy $\varepsilon_{b}$ and the defect energies $E_{c}, k^{\prime}$ depend in the same way on the concentration of cross-linking proteins (see Eqs 23). We can therefore propose that as the concentration of cross-linking proteins increases the density of defects decrease, leading to an increase in the average radius of the bundles; both the peak position and the width of the distribution (Eq7) increase with decreasing $\rho$. The more weakly bound bundles appear therefore to aggregate in a "messier" fashion, resulting in more twist-defects. The probability of exciting a defect $P_{\text {def }} \sim \exp \left(-\Delta E / k_{B} T\right)$ per unit length of filament, is higher when the energy barrier to create a defect at the bundle surface $\Delta E$, decreases with the decrease in 
the concentration of cross linkers. This prediction may be checked in future experiments.

In a living cell the actin filaments form bundles in a variety of forms. Some bundles are inside the bulk of the cell cytoplasm, and their formation may therefore resemble that of in-vitro bundles, and consequently their width may be limited by the appearance of defects. Another form of actin bundles appear in the core of stereocilia, and seem to be packed in a perfectly regular, hexagonal lattice [14]. These bundles do not form by the lateral aggregation of preexisting long filaments as in the bulk in-vitro experiments; all the filaments in such a bundle polymerize together and elongate in synchrony from the stereocilia tip. The polymerization of the filaments at their ends is promoted by tip-complex proteins. If the relative locations of the nucleation sites within this tip complex are maintained in a solid-like static structure, then the filaments which elongate from these nucleation sites will form a perfectly packed bundle. The width of these bundles is therefore not controlled by the random process of defect formation, but is very tightly controlled by the cell 14. Indeed such bundles contain thousands of actin filaments, much thicker than the in-vitro bundles
(Fig.3)

We conclude that the aggregation of long filaments by strong cross-linkers is likely to produce packing defects. Such quenched and random defects can lead to an effective pressure that limits the growth of the width of bundles, and may explain the observed peaked distribution with an exponential tail. It is a two-dimensional elastic version of the Rayleigh-instability of charged droplets [12. The rudimentary treatment given here should be improved in the future by a more rigorous treatment of the full three-dimensional problem of the complex strainfields of a population of twist-defects inside a bundle that is free to twist and bend as a whole.

\section{Acknowledgments}

I thank the Alvin and Gertrude Levine Career Development Chair, for their support. This research was supported by the Israel Science Foundation (grant No. $337 / 05)$. This research is made possible in part by the historic generosity of the Harold Perlman Family.
[1] See for example: M. L. Gardel, F. Nakamura, J. H. Hartwig, J. C. Crocker, T. P. Stossel, and D. A. Weitz, PNAS, 1031762 (2006); M.M.A.E. Claessens, M. Bathe, E. Frey and A.R. Bausch, nature materials, 5748 (2006).

[2] R. Tharmann, M.M.A.E. Claessens and A. R. Bau, Biophys. J., 902622 (2006); O. Lieleg, M.M.A.E. Claessens, C. Heussinger, E. Frey and A.R. Bausch, Phys. Rev. Lett. (2007) in press.

[3] G.H. Lai, R. Coridan, O.V. Zribi, R. Golestanian, and G.C.L. Wong, Phys. Rev. Lett. 98, 187802 (2007).

[4] I. Borukhov, R.F. Bruinsma, W.M. Gelbart and A.J. Liu, PNAS 102, 3673 (2005).

[5] T. Ikawa, F. Hoshino, O. Watanabe, Y. Li, P. Pincus and C.R. Safinya, Phys. Rev. Lett. 98, 018101 (2007).

[6] M.L. Henle and P.A. Pincus, Phys. Rev. E 71, 060801R (2005).

[7] G.M. Grason and R.F. Bruinsma, Phys. Rev. Lett. 99,
098101 (2007).

[8] Z. Shao, D. Shi and A.V. Somlyo, Biophys. J. 78950 (2000).

[9] H.J. Kwon, Y. Tanaka, A. Kakugo, K. Shikinaka, H. Furukawa, Y. Osada and J.P. Gong, Biochemistry, 4510313 (2006).

[10] J.M. Kosterlit and D.J. Thouless, J. Phys. C: Solid State Phys., 61181 (1973).

[11] L. Haviv, N.S. Gov, Y. Ideses and A. Bernheim, Euro. Biophys. J. (2007), submitted.

[12] Lord Rayleigh, Philos. Mag., 14184 (1882).

[13] A. Ott, M. Magnasco, A. Simon and A. Libchaber, Phys. Rev. E, 48 R1642 (1993).

[14] H.W. Lin, M.E. Schneider and B. Kachar, Current Opinion in Cell Biology, 1755 (2005). 
(a)
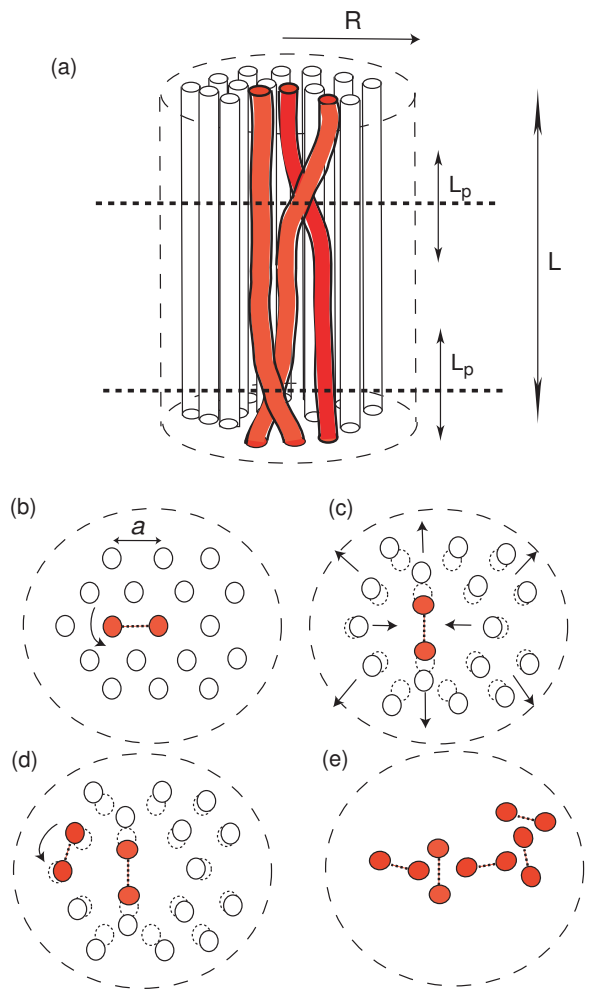

FIG. 1: (a) Schematic picture of a single twist-defect (red) inside a bundle of radius $R$ and overall length $L$. The twist extends over a length of the order of the persistence length of actin $L_{p}$. Several twist-defects along the length of the bundle cause the filaments to form a knot. Far below or above the maximal twist plane (horizontal dashed line), the filaments are arranged in a two-dimensional hexagonal array (b). (c) In the maximal twist-plane the defect (red) creates a strain field around it (arrows), and pushes the surrounding filaments from their perfectly hexagonal array (dashed circles). (d) The quadropole strain field around a defect lowers the energy to create a near-by defect with orthogonal orientation (arrow), and can result in a string-like aggregate of defects (e).
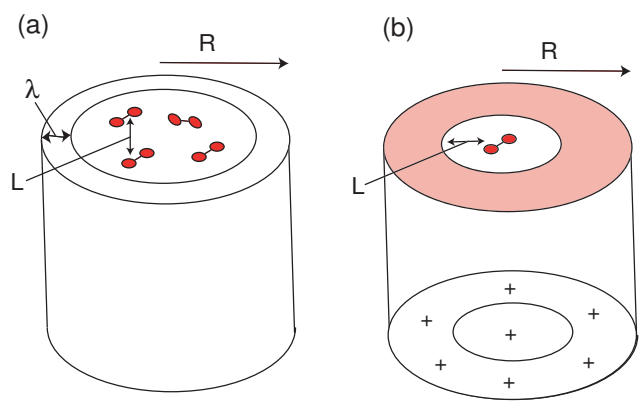

FIG. 2: (a) Schematic picture of the empty-shell model; uniform population of defects (red pairs) at areal density $\rho=1 / L^{2}$ and core energy per unit length $E_{c}$, with an empty outer shell of thickness $\lambda$. (b) Schematic calculation of the uniform field of monopoles; a single monopole (red pair in the center) interacts with a uniform distribution of neighboring monopoles of density $\rho$ (red ring), beyond a minimal separation radius $L$. This system is the elastic equivalent to a two-dimensional electrostatic repulsion problem (bottom). 


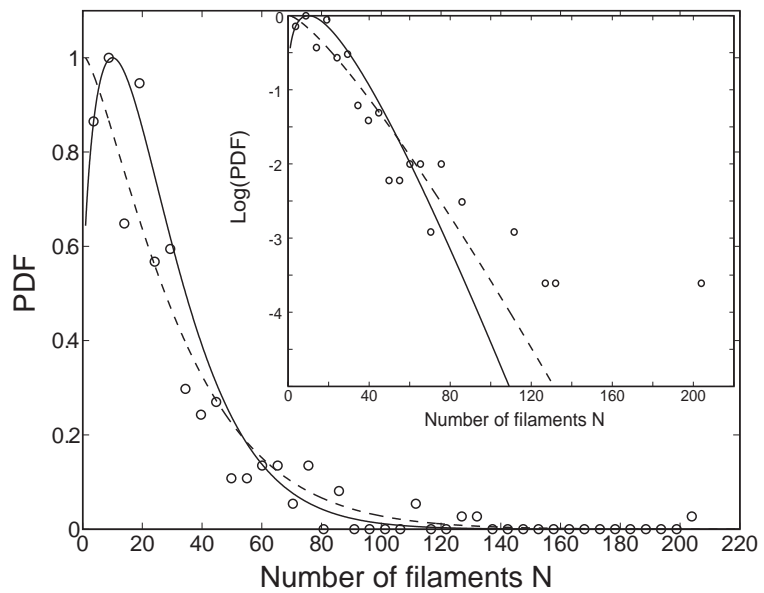

FIG. 3: Experimental distribution of the radius of actin filaments inside bundles (circles) [1], for Fascin/Actin ratio of 1:5. The calculated distribution is given by the solid line for the empty-shell model (Eq2) and dashed line for the uniform monopoles (Eq 5). The inset gives a semi-log plot of the distribution. 